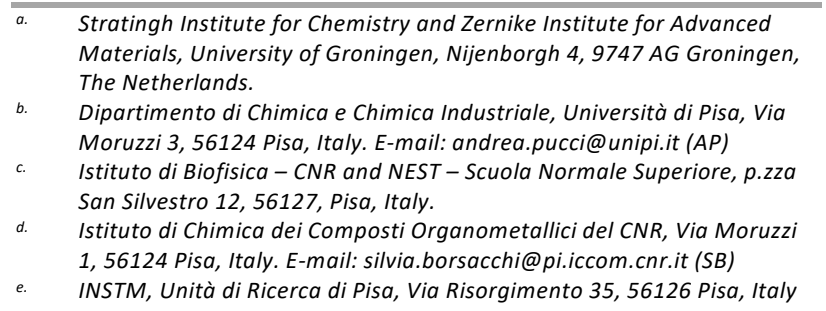

\title{
Thermochromic Polyethylene Films doped with Perylene Chromophores: Experimental Evidences and Methods for Characterization of their Phase Behaviour
}

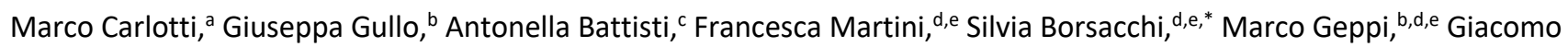
Ruggeri, ${ }^{\mathrm{b}, \mathrm{e}}$ Andrea Pucci, ${ }^{\mathrm{b}, \mathrm{e}, *}$

\begin{abstract}
We report on a thermochromic system suitable for sensing temperature changes in the $30-70{ }^{\circ} \mathrm{C}$ regime based on linear low density polyethylene (LLDPE) films doped with N,N'-bis-(1'-phenylethyl)-perylene3,4,9,10-tetracarboxydiimide (PE-Pery), a fluorescent aggregachromic dye. At low PE-Pery concentration (0.01-0.02 wt.\%), the dye monomers were well dispersed in the polymer matrix showing their maximum fluorescence intensity at $525 \mathrm{~nm}$. As the dye content was increased, monomers emission quenched whereas dyes aggregates prevailed above $0.05 \mathrm{wt} . \%$ as well as their red fluorescence band at 620-680 $\mathrm{nm}$. Upon heating from 30 to $70{ }^{\circ} \mathrm{C}$, all films displayed a thermochromic response, more evident for the less concentrated samples (<0.05 wt.\%) in which the emission of the dye as a monomer continuously increased with increasing temperature. This phenomenon promoted effective color changes from a dull red-violet at 30 ${ }^{\circ} \mathrm{C}$ to a bright yellow-green at $70{ }^{\circ} \mathrm{C}$. Combined DSC and variable-temperature Solid State NMR (SSNMR) measurements addressed the thermochromic behavior to the increased amount of available amorphous phase and to the increased mobility of both the interphase and amorphous components with temperature, which favored PE-Pery dispersion and diffusion, thus recovering their fluorescence. Overall, the present results support the use of PE-Pery-enriched LLDPE films as a chromogenic material suitable for the detection of temperature changes close to the physiological regime.
\end{abstract}

\section{Introduction}

The ability to give a prompt and distinct response is a musthave property for a functional system, especially if it has to be employed as a sensing device. In this sense, the use of fluorescence emission to monitor the variations in the properties of the observed system is quite effective. It is indeed accompanied by a characteristic response of strong intensity and it can be studied by many different parameters such as intensity, decay time, quenching efficiency and energy transfer. ${ }^{1}$

Fluorescent probes have been successfully applied as biosensors in the medical field, ${ }^{2-4}$ in devices for the detection of gasses (e.g. $\left.\mathrm{O}_{2}, \mathrm{CO}_{2}\right)^{5,6}$ and volatile organic compounds $(\text { VOCs })^{7-9}$, as $\mathrm{pH}$ and ions probes in solution ${ }^{10}$ and as active components in mechano-11-16 and thermochromic polymeric materials. ${ }^{17-20}$

Regarding the possibility to monitor temperature and mechanical stresses, aggregachromic substances offer an interesting alternative for the development of fluorescent probes. ${ }^{21-24}$ The concept of aggregachromic materials and the effects that aggregation may produce on the fluorescence emission have been already introduced; ${ }^{25}, 26$ Being driven by weak secondary interactions, the aggregation phenomenon can be easily perturbed by modifying the environmental conditions. ${ }^{12,} 13$ This idea has led to different concepts of thermochromic polymer blends based on aggregachromic dyes. Their response generally arises from local environmental changes upon temperature variations, due to phaseseparation processes for kinetically and morphologically 
controlled dye aggregation-disaggregation phenomena. ${ }^{17,18,21,}$ $24,27,28$ Polymer blends possessing kinetically controlled thermochromic properties can be obtained by dissolving a proper amount of dye in the polymer melt, followed by rapid cooling to the glassy state. ${ }^{29-31}$ Although a phase separation would be expected thermodynamically, the dye molecules are kinetically immobilized in their disaggregated form due to the high viscosity of the polymer matrix. Heating above the $\mathrm{Tg}$ yields thermodynamically stable micro/nano sized aggregates whose emission characteristics are different and mainly determined by the photophysical effects associated with the $\pi-\pi$ stacking interactions among the planar aromatic backbones of the dyes. On the other hand, morphologically controlled thermochromic effects from aggregachromic dyes While there are widespread applications of thermochromic polymers, there are scarce examples on plastic materials sensitive to physiological-range temperature changes. ${ }^{33,34}$ This Herein, we report on the thermochromic properties of polymer films based on an aggregachromic perylene diimide dye dispersed in linear low density polyethylene (LLDPE). The N,N'-bis-(1'-phenylethyl)-perylene-3,4,9,10-

tetracarboxydiimide (PE-Pery) was selected due to its wellknown aggregachromism in solution and in polyethylene, ${ }^{32}$ whereas the semi-crystalline LLDPE was chosen taking into account its broad melting endotherm, which might allow dye disaggregation also at temperature higher than $30^{\circ} \mathrm{C}$. PEPery/LLDPE films were investigated in terms of their optical response towards thermal solicitations in the range of temperatures between 30 and $70{ }^{\circ} \mathrm{C}$. The phenomenon was examined in detail by means of a combined approach based on microscopic and calorimetric techniques as well as Solid State NMR (SSNMR). More specifically, SSNMR is one of the most powerful techniques in the study of solid materials, allowing the investigation of the structural and dynamic properties of both amorphous and crystalline systems, in wide spatial (0.1$100 \mathrm{~nm}$ ) and motional ( $\mathrm{Hz}$ to $\mathrm{GHz}$ ) ranges, respectively. ${ }^{35,36}$

\section{Experimental}

\subsection{Materials}

Imidazole and linear low density polyethylene (LLDPE, Aldrich, melt flow index $190^{\circ} \mathrm{C} / 2.16 \mathrm{~kg} 1.0 \mathrm{~g} / 10 \mathrm{~min}, \mathrm{~d}=0.918 \mathrm{~g} / \mathrm{cm}^{3}$ ) were supplied by Aldrich and used without further purification. Perylene-3,4,9,10-tetracarboxy-dianhydride (PTCDA, 97\%, Aldrich) was purified as follows: $1.73 \mathrm{~g}$ of PTCDA $(4.3 \mathrm{mmol})$ were slowly added to $1.6 \mathrm{~L}$ of a hot $\left(70^{\circ} \mathrm{C}\right)$ aqueous solution of $\mathrm{NaOH} 0.3 \mathrm{M}$ in a $2 \mathrm{~L}$ Erlenmeyer flask. The solid rapidly dissolved to give a solution which was red in transmitted light, green in reflected light and emitted green fluorescence emission under $366 \mathrm{~nm}$ illumination. The solution was left under vigorous stirring for $4 \mathrm{~h}$, then was filtered on a Buckner filter. The filtrate was acidified with $6 \mathrm{M} \mathrm{HCl}$ until stable acid $\mathrm{pH}$ was reached. Precipitation of a bright red powdery solid was obtained while the solution became colorless and not fluorescent. The system was left overnight under vigorous stirring. The red solid was then filtered on a Bucker filter, washed with water until neutrality and then with acetone. The solid was then dried at $120{ }^{\circ} \mathrm{C}$ for $5 \mathrm{~h}$ and for $2 \mathrm{~h}$ at $200{ }^{\circ} \mathrm{C}$ in polymer matrices can be obtained, for example, by dissolving the dye in a semicrystalline polymer, which maintains a degree of ordered domains even above the Tg. ${ }^{32}$ In this case the chromophores are segregated from the highly ordered crystalline phase to the amorphous region, thereby causing dye molecules aggregation due to their high local concentration. By heating, polymer crystallites start to melt, thus increasing the solubility of the dye in the medium. As a consequence, the process is therefore characterized by a continuous shift of the optical properties toward the disaggregated monomeric form of the dye. Remarkably, these systems have shown to be fully reversible as the original state is recovered on cooling to ambient temperature. ${ }^{32}$

feature would be very helpful in supporting the realization of plastic devices with a wider range of application possibilities.

under vacuum $(0.1 \mathrm{mmHg}) .1 .59 \mathrm{~g}$ of red solid were recovered (yield of $92 \%$ ).

1-phenylethylamine (Aldrich) was distilled before use as follows: in a $50 \mathrm{~mL}$ Claisen apparatus with a $5 \mathrm{~cm}$ Vigreux column, $10 \mathrm{~mL}$ of 1-phenylethanamine $(\delta=0.94 \mathrm{~g} / \mathrm{mL}, 77.6$ $\mathrm{mmol})$ were added under inert atmosphere and distilled at 20 $\mathrm{mmHg}$ pressure at $230{ }^{\circ} \mathrm{C}$. The fraction distilled at $77-79{ }^{\circ} \mathrm{C}(7$ $\mathrm{mL}$ ) was recovered as pure product and kept in the dark under $\mathrm{N}_{2}$ atmosphere.

\subsection{Synthesis of $N, N$ '-bis-(1'-phenylethyl)-perylene-3,4,9,10- tetracarboxidiimide (PE-Pery)}

In a $250 \mathrm{~mL}$ flask equipped with a condenser, $1.448 \mathrm{~g}$ of PTCDA (3.6 mmol), $1.16 \mathrm{~mL}$ of freshly distilled 1-phenylethylamine $(\delta$ $=0.94 \mathrm{~g} / \mathrm{mL}, 9.6 \mathrm{mmol}$ ) and $30 \mathrm{~g}$ of imidazole were introduced under inert $\left(\mathrm{N}_{2}\right)$ atmosphere and the system was warmed up to $180{ }^{\circ} \mathrm{C}$ and stirred for $4 \mathrm{~h}$. The crude product was then transferred in a $500 \mathrm{~mL}$ Erlenmeyer flask with the help of 130 $\mathrm{mL}$ of boiling water. After cooling, $240 \mathrm{~mL}$ of $\mathrm{HCl} 2 \mathrm{M}$ were added and the system left overnight under vigorous stirring. The resulting dark-red solid was filtered off, washed thoroughly with distilled water until the $\mathrm{pH}$ of washings turned to be neutral and dried at $200{ }^{\circ} \mathrm{C}$ under vacuum $(0.1 \mathrm{mmHg})$ for $5 \mathrm{~h}$.

The crude product was purified by chromatography on silica gel using chloroform/ethyl acetate $20: 1$ by vol as eluent; $0.99 \mathrm{~g}$ of red needle crystals were recovered (yield: $57.0 \%$, Figure $\mathrm{S} 1)$.

${ }^{1} \mathrm{H}$ NMR $\left(\mathrm{CDCl}_{3}, 25^{\circ} \mathrm{C}\right): \delta=8.3\left(\mathrm{~d}, \mathrm{~J}(\mathrm{H}, \mathrm{H})=7.9 \mathrm{~Hz} 4 \mathrm{H} ; \mathrm{CH}_{2}\right) ; 8.0$ $\left(\mathrm{d}, \mathrm{J}(\mathrm{H}, \mathrm{H})=8.1 \mathrm{~Hz} 4 \mathrm{H} ; \mathrm{CH}_{2}\right) ; 7.3\left(\mathrm{~m}, 5 \mathrm{H} ; \mathrm{CH}_{2}\right) ; 6.5(\mathrm{q}, \mathrm{J}(\mathrm{H}, \mathrm{H})=$ $6.9 \mathrm{~Hz} 2 \mathrm{H} ; \mathrm{CH}), 2.0\left(\mathrm{~d}, \mathrm{~J}(\mathrm{H}, \mathrm{H})=7.1 \mathrm{~Hz} 6 \mathrm{H} ; \mathrm{CH}_{3}\right) \mathrm{ppm}$;

${ }^{13} \mathrm{C} \mathrm{NMR}\left(\mathrm{CDCl}_{3}, 25{ }^{\circ} \mathrm{C}\right): \delta=163.0(\mathrm{CO}), 140.0$ (2C; benzene), 134.4 (4C; perylene), 130.9 (4C; perylene), 128.5 (4C; benzene $+8 \mathrm{C}$; perylene), 126.7 (2C; perylene $+6 \mathrm{C}$; benzene), 123.5 (2C; perylene), 51.2 ( $\mathrm{CH}$ aliph), $17.7\left(\mathrm{CH}_{3}\right) \mathrm{ppm}$;

FTIR (KBr): $u=3093$ (uCH arom.), 2964, 2938, (uCH aliph), 1697, 1657, 1593, 1577 (uCO imide), 1503, 1438, 1395, 1340 (uCC ring) $\mathrm{cm}^{-1}$;

\subsection{Preparation of PE-Pery/LLDPE films}


$0.80 \mathrm{~g}$ of LLDPE were dissolved in $65 \mathrm{~mL}$ of toluene at $100{ }^{\circ} \mathrm{C}$ for about $4 \mathrm{~h}$. Then, the proper amount of dye was added from a $0.5 \mathrm{mg} / \mathrm{mL}$ chloroform solution. The solvent was then removed under reduced pressure $(20 \mathrm{mmHg})$ and the obtained blend dried under heating $\left(100{ }^{\circ} \mathrm{C}\right)$ at $0.1 \mathrm{mmHg}$ for $5 \mathrm{~h} .0 .5 \mathrm{~g}$ of the dry blend was then cut into small pieces and meltpressed between two Teflon foils in a Carver 3851-0 press at 4.5 ton at $160{ }^{\circ} \mathrm{C}$ for $3 \mathrm{~min}$. Various cutting-pressing cycles were performed in order to obtain homogeneous films of about $100 \mu \mathrm{m}$ of thickness. Annealing experiments were performed by placing the films on a temperature controlled hot stage in the range of temperature $30-50{ }^{\circ} \mathrm{C}$.

\subsection{Characterization}

NMR measurements in solution were performed on a spectrometer operating at 600 and $150 \mathrm{MHz}$ for ${ }^{1} \mathrm{H}$ and ${ }^{13} \mathrm{C}$, respectively, and the temperature was controlled to $\pm 0.1{ }^{\circ} \mathrm{C}$. All ${ }^{1} \mathrm{H}$ and ${ }^{13} \mathrm{C}$ NMR chemical shifts are referenced to tetramethylsilane as external standard using $\mathrm{CDCl}_{3}$ as solvent. Infrared spectra were recorded by a Fourier transform infrared spectrometer (Spectrum One, PerkinElmer) on $\mathrm{KBr}$ windows. UV-Vis spectra of THF solutions were recorded at room temperature in isotropic conditions with a Perkin Elmer Lambda 650.

Fluorescence spectra were recorded at room temperature with the help of a Horiba Jobin-Yvon Fluorolog ${ }^{\circledR}-3$ spectrofluorometer equipped with F-3000 Fiber Optic Mount plus fiber-optic bundles.

Microscopy images and lifetime measurements were collected by using a Leica TCS SP5 SMD inverted confocal microscope (Leica Microsystems AG, Wetzlar, Germany) equipped with an external pulsed diode laser (PicoQuant $\mathrm{GmbH}$, Berlin, Germany) for excitation at $405 \mathrm{~nm}$. The laser repetition rate was set to be $40 \mathrm{MHz}$. Each of the image sizes were $512 \times 512$ pixels and acquired with a scan speed of $400 \mathrm{~Hz}$ (lines per second). The pinhole aperture was set at 1.00 Airy. PEPery/LLDPE films fixed on microscope glass slides were viewed with a $100 \times 1.3 \mathrm{NA}$ oil immersion objective (Leica Microsystems). The images were collected using low excitation power at the sample (10-20 $\mu \mathrm{W})$. Emissions were monitored in the $430-490 \mathrm{~nm}$ range by acousto-optical tuneable beam splitter (AOBS) based built in detectors. Acquisition lasted until about 100-200 photons per pixel were collected, at photon counting rates of 100-500 kHz. Emission lifetime images (FLIM) of the PE-Pery/LLDPE films were elaborated using Picoquant Symphotime software for FLIM analysis.

The thermal behavior was evaluated by differential scanning calorimetry (DSC) under nitrogen atmosphere by using a Mettler Toledo StarE System, equipped with a DSC822C module. Films were heated from 25 to $150{ }^{\circ} \mathrm{C}$ at $10^{\circ} \mathrm{C} / \mathrm{min}(1 \mathrm{st}$ heating), cooled to $25^{\circ} \mathrm{C}$ at the same scan rate (1st cooling), then heated again to $150{ }^{\circ} \mathrm{C}$ at $10{ }^{\circ} \mathrm{C} / \mathrm{min}$ (2nd heating). Melting enthalpies were evaluated from the integrated areas of melting peaks by using indium for calibration. PE crystalline content $(X)$ was evaluated from the measured melting enthalpy $\left(\Delta \mathrm{H}_{m}\right)$ taking into account the melting enthalpy of the perfect PE crystal $\left(\Delta \mathrm{H}_{\mathrm{m}}^{\circ}, 277.1 \mathrm{~J} / \mathrm{g}\right)$, using equation 1:

(eq. 1)

${ }^{13} \mathrm{C}$ Cross Polarization SSNMR spectra were recorded on a dualchannel Varian InfinityPlus 400 spectrometer, equipped with a $3.2 \mathrm{~mm}$ Cross Polarization/Magic Angle Spinning (CP/MAS) probehead, working at $400.03 \mathrm{MHz}$ for proton and at 100.61 $\mathrm{MHz}$ for carbon-13, with ${ }^{1} \mathrm{H}$ and ${ }^{13} \mathrm{C}$ pulse durations of $3 \mu \mathrm{s}$. All the spectra were acquired using a recycle delay of $5 \mathrm{~s}$, a contact time of $5 \mathrm{~ms}$, and a MAS frequency of $8 \mathrm{kHz}$. Spectra were recorded varying the temperature from 20 to $90{ }^{\circ} \mathrm{C}$ and letting the temperature to equilibrate for 10 minutes before performing the experiment.

A 2D Wideline Separation Experiment (WISE) was carried out using a MAS frequency of $3.5 \mathrm{kHz}$, a contact time of $0.1 \mathrm{~ms}$ and a recycle delay of $5 \mathrm{~s}$.

TMS was used as a primary chemical shift reference for all nuclei, while hexamethylbenzene was the secondary reference for ${ }^{13} \mathrm{C}$.

On-resonance ${ }^{1 \mathrm{H}}$ Free Induction Decays (FIDs) were recorded on a spectrometer made of a Stelar PC-NMR system and a permanent magnet, providing a magnetic field corresponding to a Larmor frequency of $20 \mathrm{MHz}$, using a ${ }^{1} \mathrm{H}$ pulse duration of $4 \mu \mathrm{s}$. A solid-echo pulse sequence with an echo delay of $12 \mu \mathrm{s}$ was used, accumulating 200 transients, with a recycle delay of $3 \mathrm{~s}$. ${ }^{1} \mathrm{H}$ FIDs were recorded by progressively increasing the temperature from 25 to $100{ }^{\circ} \mathrm{C}$, and letting the temperature to equilibrate for 10 minutes before the experiment. All the variable temperature measurements were carried out using air as heating gas.

\section{Results and discussion,}

\subsection{Effect of PE-Pery concentration on the optical properties of LLDPE films}

Linear low-density polyethylene (LLDPE) films containing different concentrations of PE-Pery (0.01-0.5 wt.\%) were prepared by compression molding of the respective dye/LLDPE mixtures. Various cutting-pressing cycles were performed in order to obtain homogeneous films of about $100 \mu \mathrm{m}$ of thickness. It has been reported that perylene chromophores dispersed in PE start to aggregate above a certain concentration into supramolecular assemblies, which usually have optical properties different from the isolated monomer. ${ }^{37}$ Aggregachromic dyes incorporated into polymers as thermodynamically stable micro-/nano-sized aggregates of a few molecules show optical properties, which derive mainly from the absorption or the fluorescence emission of chromophores interacting through $\pi-\pi$ stacking forces among their planar aromatic backbones. Dye aggregates exhibit distinct changes in the absorption band as compared to the monomeric species; the bathochromically shifted J-bands and 
hypsochromically shifted $\mathrm{H}$-bands of the aggregates (in a ladder-, staircase- or brickwork-arrangement) have been explained in terms of molecular exciton coupling theory, i.e., coupling of transition moments of the constituent dye molecules. ${ }^{37}$ Accordingly, aggregation of PE-Pery dyes in LLDPE films showed to affect their absorption properties. Besides the typical $0-0$ and $0-1 \pi-\pi^{*}$ transitions of the isolated noninteracting chromophores at 524 and $490 \mathrm{~nm}$, an unstructured band centered at $570 \mathrm{~nm}$ emerged upon increasing PE-Pery concentration, possibly attributed to the formation of $\mathrm{J}$ aggregates (Figure 1a). LLDPE films containing lower concentrations of PE-Pery (0.01-0.02 wt.\%) displayed the typical emission features of the $0-0$ and $0-1$ emission bands at about. Analogously to UV-vis experiments, the increase of dye concentration (0.05-0.5 wt.\%) promotes the electronic coupling between perylene chromophores, which induced emission quenching of the isolated dyes and the progressive evolution of the aggregate band from 600 to $650 \mathrm{~nm}$ (Figure 1b). Notably, the existence of a well-defined isosbestic point centered at $590 \mathrm{~nm}$ clearly indicated the presence of two states assigned to the aggregated and the monomeric forms of PE-Pery.
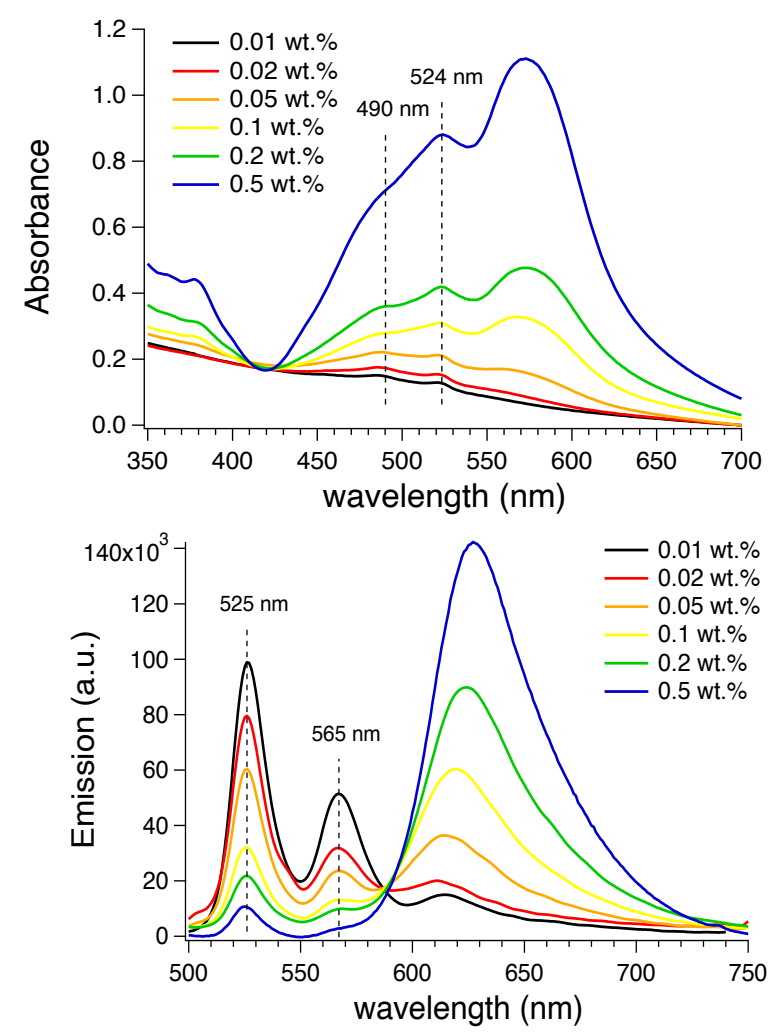

(a)

(b)

Figure 1. Absorption (a) and fluorescence (b) spectra ( $\lambda$ exc. $=450 \mathrm{~nm}$ ) of PEPery/LLDPE films at different PE-Pery concentration.

Emission imaging studies were carried out investigating the morphology of PE-Pery/LLDPE films as a function of dye concentration. In more detail, the aggregation pattern of PEPery in the LLDPE films was analyzed by means of a confocal scanning laser fluorescence microscope (CSLM). The fluorescence of PE-Pery in the films was excited by a laser source at $458 \mathrm{~nm}$. High-resolution fluorescence images of the same film area were concomitantly taken in two wavelength intervals: $500-570 \mathrm{~nm}$ (Figure 2, column 1, fluorescence in green), where PE-Pery monomer has prevalent emission, and 620-680 nm (Figure 2, column 2, fluorescence in red), where aggregated PE-Pery has prevalent emission (note that images are in pseudocolors). At low PE-Pery concentration (0.01 wt.\%, Figure $2 \mathrm{a}$ ), the monomeric dye emission is clearly visible in the 500-570 $\mathrm{nm}$ channel, whereas the aggregation pattern of PEPery is completely absent in the $620-680 \mathrm{~nm}$ channel. Conversely, the complex texture of PE-Pery assembly appears visible at higher concentration $(0.2 \mathrm{wt} . \%$, Figure $2 \mathrm{~b})$ : upon selfassembly, the dye yields phase-separated microcrystal structures with dimensions in the range of a few micrometers that are randomly dispersed in the film matrix. Interestingly, the background matrix contains a very small amount of PEPery in the monomeric form, as clearly displayed by the poor fluorescence in the 500-570 $\mathrm{nm}$ channel.
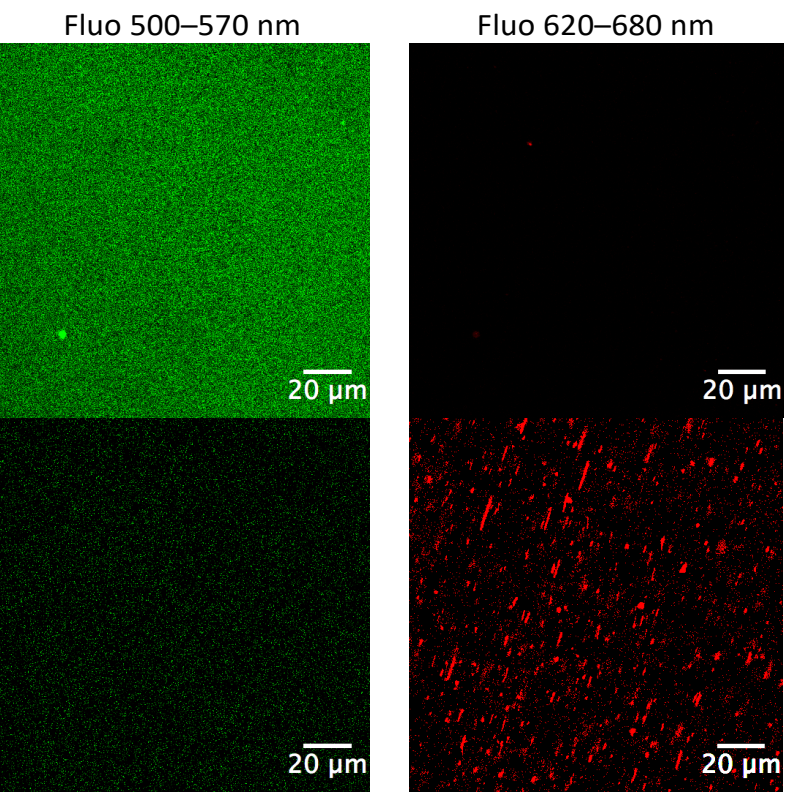

(a)

Figure 2. Confocal microscope images of PE-Pery/LLDPE films. First column: fluorescence images collected in the $500-570 \mathrm{~nm}$ range for films containing (a) 0.01 wt.\% and (b) 0.2 wt.\% of PE-Pery, respectively; second column: fluorescence images collected in the 620-680 nm range for films containing the (a) 0.01 wt.\% and (b) 0.2 wt.\% of PE-Pery, respectively. Note that images are in pseudocolors

The quenching of fluorescence as a consequence of dye concentration is well documented both for solutions and solid matrices. ${ }^{38-40}$ The formation of quenching centres can arise from diffusion of the excited fluorophores within their lifetime or by means of energy transfer between closely spaced pairs. The former better describes the solution state where high diffusion coefficients exist, whereas the latter is more suited for rigid matrices. ${ }^{38}$ The experimental findings here reported support the existence of the following mechanism: at low PEPery concentration, the dye monomers are well dispersed in the polymer matrix therefore showing their maximum fluorescence intensity; next, as the dye content is increased 
fluorescence starts to decrease being higher the probability to find quenching centres (in our case, for PE-Pery concentration comprised in the range 0.01-0.05 wt.\%); finally, above a concentration threshold (i.e., 0.05 wt.\%), aggregates of dye begin to form in large amount and the red fluorescence band starts to be predominant.

\subsection{Effect of temperature on the optical properties of PE- Pery/LLDPE films}

PE-Pery/LLDPE films which showed a noticeable fluorescence decrease with increasing dye content (i.e., PE-Pery concentration $\geq 0.02$ wt.\%), were thermally stressed at temperatures ranging from about $30{ }^{\circ} \mathrm{C}$ to $70{ }^{\circ} \mathrm{C}$, by placing them in contact with a thermostatically controlled $\left( \pm 0.1{ }^{\circ} \mathrm{C}\right)$ metal surface. The effect provided by temperature changes was evaluated by means of fluorescence spectroscopy by collecting emission spectra about $15 \mathrm{~s}$ after the temperature increase to ensure film relaxation to a new thermal equilibrium. The thermochromic behaviour was also evaluated in terms of fluorescence intensity variation of the monomer emission band at $525 \mathrm{~nm}$ with temperature. Upon heating from 30 to $70{ }^{\circ} \mathrm{C}$, all PE-Pery/LLDPE films resulted affected by temperature changes. Notably, the $0-0$ and $0-1$ transitions at about 525 and $565 \mathrm{~nm}$ start to significantly increase already at annealing temperatures as high as $35{ }^{\circ} \mathrm{C}$, together with the progressive recovery of the overall fluorescence intensity (Figure 3).
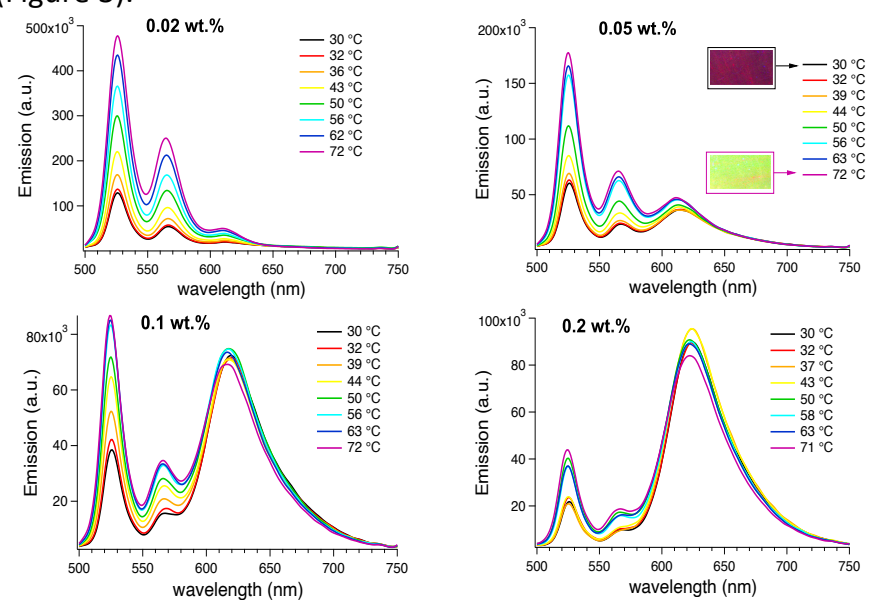

Figure 3. Fluorescence spectra $\left(\lambda_{\text {exc. }}=450 \mathrm{~nm}\right.$ ) of PE-Pery/LLDPE films as a function of the annealing temperature. PE-Pery concentration: up left, 0.02 wt.\%; up right, 0.05 wt.\%; down left, $0.1 \mathrm{wt} . \%$; down right, $0.2 \mathrm{wt} . \%$. For the film containing the 0.05 wt.\% of PE-Pery pictures taken under illumination at $366 \mathrm{~nm}$ at $30^{\circ} \mathrm{C}$ (black box) and $70^{\circ} \mathrm{C}$ (purple box) are reported as insets

The most evident thermochromic response was found for the 0.05 wt.\% PE-Pery/LLDPE film, where the largest variation of the fluorescence intensity gives rise to effective colour changes from red-violet at $30{ }^{\circ} \mathrm{C}$ to yellow-green at $70{ }^{\circ} \mathrm{C}$ (Figure 3, insets). Moreover, once the heating was removed the film restored rapidly the original optical properties previously recorded at $30{ }^{\circ} \mathrm{C}$, suggesting a complete reversibility of the phenomenon (Figure S2). It is worth to notice that the relative variation of the fluorescence intensity at $525 \mathrm{~nm}$ (i.e., $\left(I-I_{0}\right) / I_{0}$, where $I_{0}$ is the emission of the film at $30{ }^{\circ} \mathrm{C}$ ) becomes progressively less pronounced as the content of the dye increases, and its maximum shifts to lower temperature, i.e. from about $70{ }^{\circ} \mathrm{C}$ for the 0.02 wt.\% to $50{ }^{\circ} \mathrm{C}$ for the $0.2 \mathrm{wt} . \%$ (Figure 4).

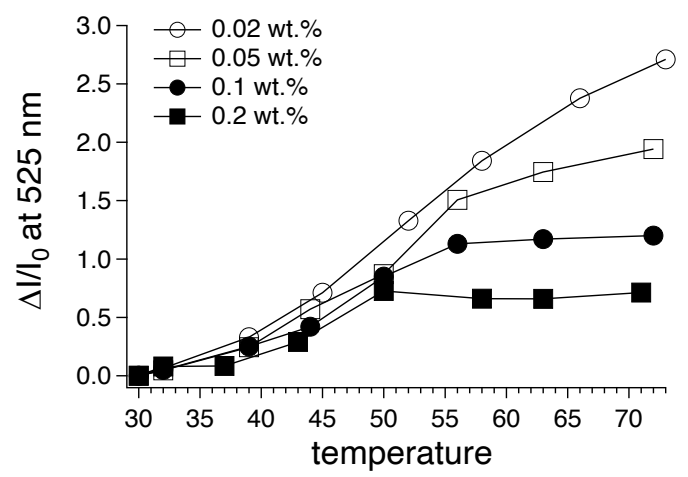

Figure 4. Plot of the $\left(I-I_{0}\right) / I_{0}$ ratio calculated for the peak at $525 \mathrm{~nm}$ as a function of temperature for different PE-Pery/LLDPE films

Notably, the temperature increase does not cause significant variations of the emission intensity of the red-shifted aggregate band. In stark contrast, the fluorescence intensity at $525 \mathrm{~nm}$ is strongly impacted upon temperature changes. These findings support the hypothesis that during heating, the different solubility of PE-Pery in the polymer and the increased mobility of the macromolecules of LLDPE in the amorphous phase help in keeping dye monomers at a distance at which they do not interact with each other. It is possible that the thermochromic behavior could be mostly ascribed to the ability of the monomers to diffuse within the polymer bulk at higher temperature, therefore increasing their quantum efficiency and limiting the formation of quenching centers. Conversely, the PE-Pery aggregates, that are still present even at low concentrations of dye in the polymer and which originate the red-shifted aggregate band, seem mostly unaffected by temperature changes.

\subsection{Investigation of the phase properties of PE-Pery/LLDPE films by DSC and SSNMR}

To validate our hypothesis, we probed the thermal properties of PE-Pery/LLDPE films containing different amounts of dye. The crystallinity of three LLDPE samples with 0 (neat polymer), 0.05 and 0.5 wt.\% of PE-Pery was investigated by comparing their DSC thermograms (see for example in Figure S3 the DSC traces of the 0.05 wt.\% PE-Pery/LLDPE film). The crystalline fraction was estimated as the ratio between the experimental enthalpy of melting and the theoretical value calculated for $100 \%$ crystalline PE $\left(\Delta H_{0}=277.1 \mathrm{~J} / \mathrm{g}\right){ }^{41}$ The obtained results are reported in Figure 5. 


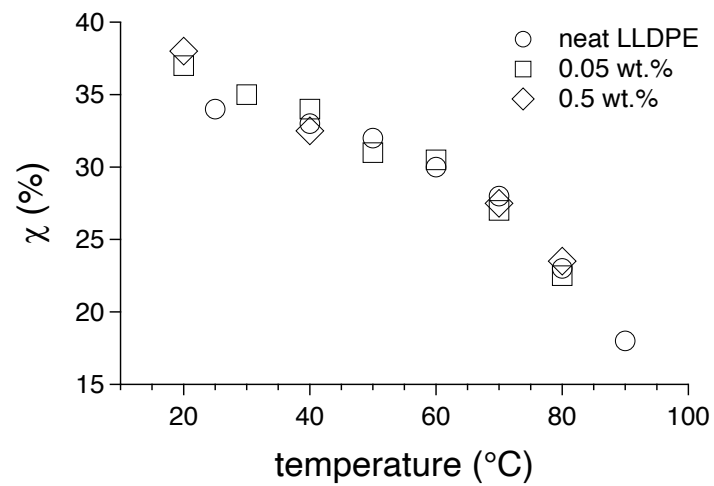

Figure 5. Crystallinity of neat LLDPE and PE-Pery/LLDPE films as calculated by DSC

No apparent modification of the crystalline contents were found in all LLDPE films upon the addition of PE-Pery. Notably, upon heating above $30-40{ }^{\circ} \mathrm{C}$ the crystalline fraction of LLDPE decreased progressively with a sharp decline just after 60$70{ }^{\circ} \mathrm{C}$. This phenomenon matches well with the rise in fluorescence of the PE-Pery/LLDPE films, which steepened their emission recovery above $40{ }^{\circ} \mathrm{C}$. These results support the idea that the larger is the fraction of available amorphous phase, the higher is the diffusion rate of PE-Pery within the LLDPE matrix, thus preventing the formation of closely spaced quenching centers that lessen the overall fluorescence intensity. Moreover, a larger fraction of amorphous phase means also a larger volume available for dye dispersion in a molecularly dissolved fashion. On the other hand, the decrease of crystallinity with increasing temperature seems to be substantially the same for all samples, while the fluorescence emission properties at variable temperature appeared clearly dependent on the dye concentration.

With the aim of going more in depth into the investigation of the variation with temperature of the phase properties of these samples, we applied several Solid State NMR techniques, which are very powerful in clarifying these aspects.

In Figure 6 the ${ }^{13} \mathrm{C}$ CP-MAS spectra of LLDPE containing 0.5 wt.\% of PE-Pery recorded in the temperature range $30-80{ }^{\circ} \mathrm{C}$ are reported. It is immediately evident that LLDPE gives rise to a structured signal which, as proved by the spectral fitting reported in Figure 7, is constituted by three peaks, centred at about 31, 32 and 33 ppm, ascribable to LLDPE amorphous, interphase and crystalline domains, respectively (signals of PEPery could not be observed due to its very low concentration). With the term interphase we here refer to that intermediate phase, whose existence has been stated since long time, ${ }^{42}$ between crystalline domains, where polymer chains are very much ordered and experience very scarce mobility and amorphous regions characterized by complete disorder and large chain mobility. Polymer chains in the interphase experience a restricted mobility and a quite disordered arrangement. ${ }^{13} \mathrm{C}$ CP-MAS spectra are intrinsically not quantitative so information on the absolute amounts of the different phases cannot be straightforwardly extracted. However it is evident that, as expected, with increasing temperature the intensity of the peak of the amorphous phase increases and its linewidth decreases, which is due to a progressive amorphization of the polymer and an increasing mobility of its chains.

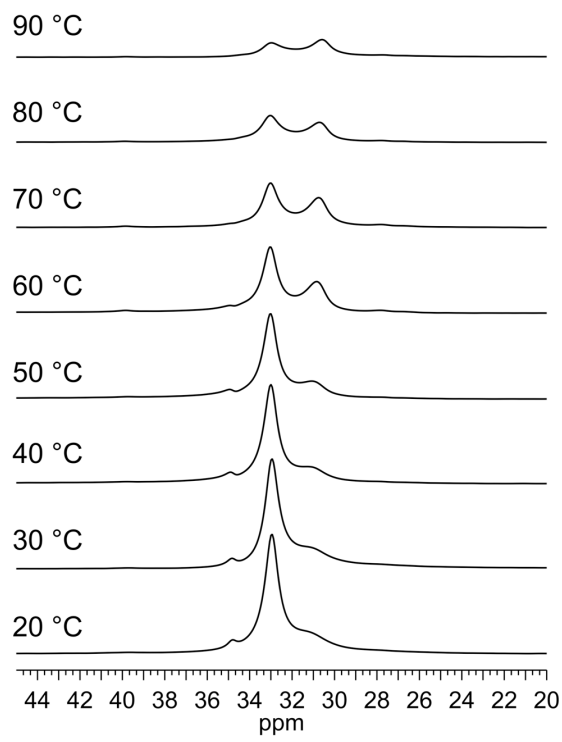

Figure $6 .{ }^{13} \mathrm{C}$ CP-MAS spectra of LLDPE containing 0.5 wt.\% of PE-Pery registered in the temperature range $20-90{ }^{\circ} \mathrm{C}$

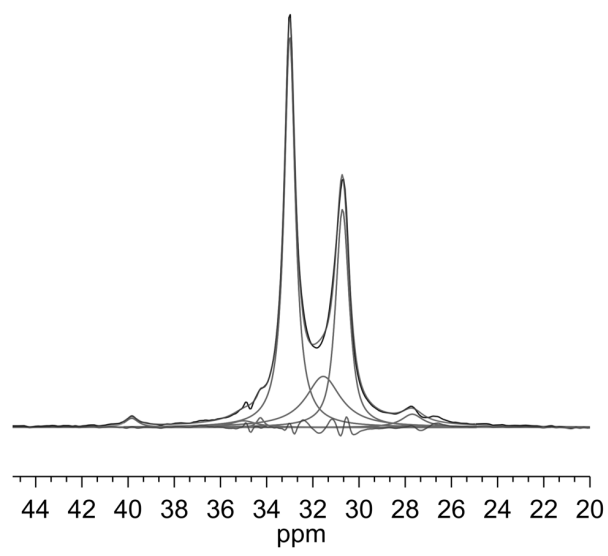

Figure 7. Spectral fitting of the ${ }^{13} \mathrm{C} C P$-MAS spectrum of LLDPE containing 0.5 wt.\% of PE-Pery registered at $70^{\circ} \mathrm{C}$. The three peaks, from lower to higher chemical shift, are those of the amorphous, interphase and crystalline domains

More detailed as well as quantitative information on the phase properties of LLDPE in the different samples could be obtained from the analysis of the ${ }^{1} \mathrm{H}$ Free Induction Decays (FIDs), recorded in the temperature range $20-100{ }^{\circ} \mathrm{C}$. In the presence of a low external magnetic field, ${ }^{1} \mathrm{H}$ FIDs of solid samples can be recorded under on-resonance conditions and reproduced, by a fitting procedure, with a linear combination of analytical functions $\left(f_{i}\right)$, each characterized by a spin-spin relaxation time $T_{2 i}$ and a weight percentage, $w_{i} . T_{2 i}$, out of the so called rigid lattice regime, monotonically increases with increasing molecular mobility, while $w_{i}$ approximately corresponds to the percentage of ${ }^{1} \mathrm{H}$ nuclei of the sample whose signal is represented by the $i$-th function. ${ }^{43-46}$ The linear combination of 
functions best reproducing the experimental FID is chosen on the basis of the Occam's Razor principle and of the minimization of the $\chi^{2}$ of the fitting, while $T_{2 i}$ and $w_{i}$ are obtained as fitting parameters.

${ }^{1} \mathrm{H}$ FIDs were recorded and analyzed for pure LLDPE and for LLDPE containing $0.02,0.05$ and 0.5 wt.\% of PE-Pery. In all cases the best fitting linear combination of functions (chosen among Pake, Abragamian, Gaussian, Weibullian, and exponential) for reproducing the experimental FIDs included a Pake, a Gaussian and an exponential, which, on the basis of their nature, $T_{2}$ values and literature results ${ }^{47}$ can be ascribed to crystalline, interphase and amorphous LLDPE domains, respectively. In Figure 8 the fitting of the FID of the sample containing 0.02 wt.\% of PE-Pery at $50{ }^{\circ} \mathrm{C}$ is reported as an example.

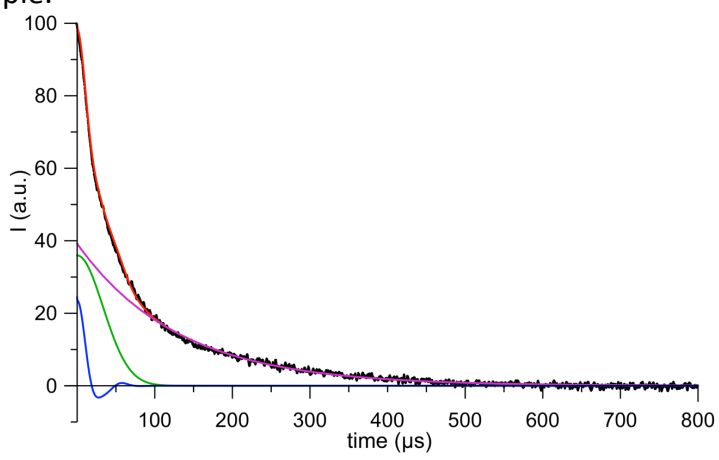

Figure $8 .{ }^{1} \mathrm{H}$ FID analysis of the LLDPE sample containing $0.02 \mathrm{wt} . \%$ of PEPery at $50{ }^{\circ} \mathrm{C}$. The fitting function is plotted in red, while in blue, green and magenta its Pake, Gaussian and exponential components are shown, respectively.

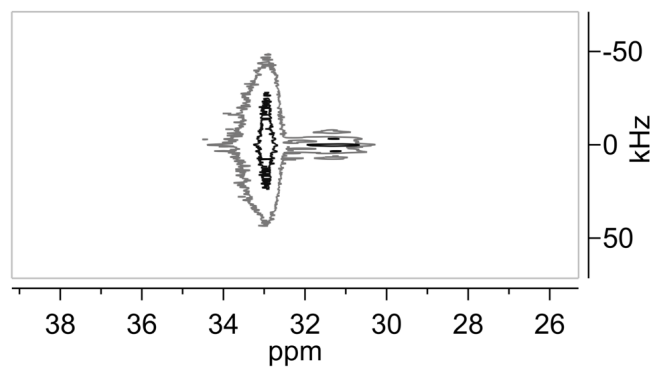

(a)

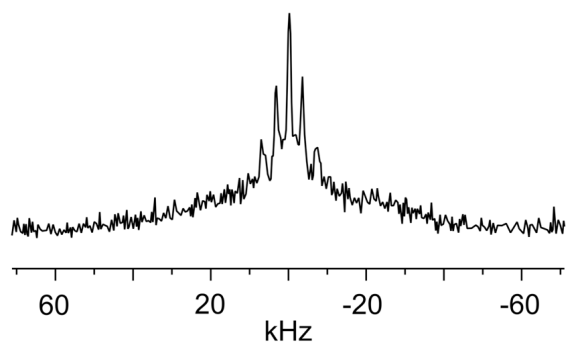

(b)

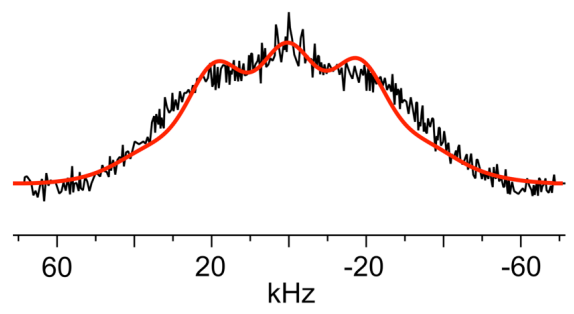

(c)

Figure 9. (a) $2 \mathrm{D}^{1} \mathrm{H}-{ }^{13} \mathrm{C}$ WISE map of LLDPE; (b) ${ }^{1} \mathrm{H}$ pattern corresponding to the ${ }^{13} \mathrm{C}$ peak at $31 \mathrm{ppm}$; (c) ${ }^{1} \mathrm{H}$ experimental pattern corresponding to the ${ }^{13} \mathrm{C}$ peak at $33 \mathrm{ppm}$ (black) and simulated pattern corresponding to the Fourier Transformation of a combination of the Gaussian and Pake functions used in the ${ }^{1} \mathrm{H}$ FID analysis

This assignment could also be confirmed by $2 \mathrm{D}{ }^{1} \mathrm{H}-{ }^{13} \mathrm{C}$ WISE experiment, that provides a $2 \mathrm{D}$ spectrum in which ${ }^{13} \mathrm{C}$ isotropic peaks are correlated with the quasi-static signal of the coupled ${ }^{1} \mathrm{H}$ nuclei. ${ }^{48}$ In Figure 9 the 2D WISE map and the ${ }^{1} \mathrm{H}$ signals corresponding to the ${ }^{13} \mathrm{C}$ peaks at 31 and $33 \mathrm{ppm}$ are shown. It is possible to observe that the ${ }^{13} \mathrm{C}$ peak of amorphous LLDPE is correlated with a signal constituted by a narrow central peak and several spinning sidebands, which corresponds to the exponential function of the ${ }^{1} \mathrm{H}$ FID. On the other hand, the ${ }^{13} \mathrm{C}$ peak of the crystalline phase at $33 \mathrm{ppm}$ is correlated with a broad signal that can be well reproduced with a combination of a Pake and a Gaussian lineshape, characterized by the same parameters found from FID analysis. The presence of the Gaussian component is probably due to the fact that the interphase gives rise to a weak and broad ${ }^{13} \mathrm{C}$ signal strongly overlapped with those of the crystalline phase. Moreover it can be observed that the ${ }^{1} \mathrm{H}$ signal mainly attributed to the crystalline/interphase domains contains a small contribution from the ${ }^{1} \mathrm{H}$ signal of the amorphous phase, and viceversa, which is due to the strong overlap between the ${ }^{13} \mathrm{C}$ signals. 
Useful information can be obtained by looking at the trends with temperature of $T_{2}$ and $w$ of the three functions obtained from the analysis of the FIDs of the four different samples considered.

Figure 10a shows the trend of the weight percentage of the Pake function with temperature, which somehow corresponds to LLDPE crystallinity, together with crystallinity data obtained by DSC. First, it can be noticed that the crystallinity as determined by DSC is systematically higher than the percentage of the Pake function. This is probably due to a contribution to the melting enthalpy from the quite rigid interphase, which is separately considered in the NMR analysis (i.e. described by the Gaussian function). On the other hand it is interesting to observe that, even if the weight of the Pake function for all samples decreases with increasing temperature, as expected due a progressive approach to the melting, the values found for pure LLDPE are systematically, even if slightly, lower than those of the samples containing PEPery, among which no significant differences can be observed. This result suggests that PE-Pery could act as nucleant, so favoring the formation of LLDPE crystalline domains during the cooling from the melt. This difference between LLDPE and the dyed samples, not detected by DSC, could be highlighted by SSNMR analysis probably because of its ability to reveal very small, i.e. nanometric sized, crystalline domains, not detectable by DSC.

It is generally accepted that in semicrystalline polymers the organic dye is excluded from the crystalline regions, while it can be found at the interface between amorphous and crystalline phases and in the amorphous phase. ${ }^{11},{ }^{13}$ In agreement with this, the parameter of the Pake function, which is related to the inverse of its $T_{2}$, shows a slightly decreasing trend with increasing temperature (figure not shown), due to a slow progressive increase of mobility of LLDPE chains in the crystalline domains, which is independent of the dye concentration.

Interesting information can be obtained by looking at $w$ and $T_{2}$ of the Gaussian function, describing the interphase between LLDPE crystalline and amorphous domains (Figures 10b and 10c). At temperatures lower than $60{ }^{\circ} \mathrm{C}$ the weight of the Gaussian function is higher in the FIDs of the dyed samples, with respect to pristine LLDPE, indicating the presence of a larger amount of interphase, that appears substantially independent of the dye concentration. It is possible that, on cooling from the melt, a fraction of dye monomers remains entrapped in the interphase, somehow favoring the formation of this rigid and disordered phase. On the other hand, it is quite unlikely that bulkier dye aggregates could be hosted in the interphase. In the two less concentrated samples the amount of interphase slightly decreases with increasing temperature, with a corresponding increase of the amorphous phase. For pristine LLDPE the decrease of interphase with increasing temperature is clearly larger. The behavior of the sample with $0.5 \mathrm{wt} . \%$ of PE-Pery resembles that of the more diluted samples below $60{ }^{\circ} \mathrm{C}$, while above this threshold it becomes similar to that of LLDPE. A possible interpretation is that in this sample a dye amount larger than that thermodynamically allowed remains kinetically enclosed in the interphase: when the temperature is increased above $60^{\circ} \mathrm{C}$ thermodynamics prevails and the amount of interphase exceeding that of pristine LLDPE is quickly destroyed, thus releasing the dye in the amorphous phase.

$T_{2}$ of the Gaussian function shows, for all samples, an increasing trend with increasing temperature, indicating that the mobility of LLDPE chains in the interphase increases with increasing temperature. It must be noticed that for all the dyed samples $T_{2}$ is systematically larger than for pristine LLDPE, and does not show a significant dependence on PE-Pery concentration. This can be understood considering that PEPery acts as a "defect" in the interphase, which disturbs the usual enthalpically favored interactions among polymer chains, that are moved apart in order to minimize the interactions with the defect. Therefore a free volume forms around the defect, in which the polymer chains mobility is favored. ${ }^{49}$

Figure $10 \mathrm{~d}$ shows the behavior of $T_{2}$ of the exponential function, representing LLDPE amorphous phase, with increasing temperature. It can be observed that $T_{2}$, and therefore the mobility of LLDPE chains in the amorphous regions, increases with increasing temperature, and it is larger for higher PE-Pery concentration. This can be explained again considering that the dye is responsible for the creation of free volume in which the polymer mobility is favored.

If now we consider again the fluorescence emission properties at variable temperature, the phase properties just highlighted by SSNMR provide some possible explanations of the differences among samples with different dye concentration. It was observed that for the less concentrated samples (0.02 and 0.05 wt.\%) the emission of the dye as a monomer continuously increased with increasing temperature, while for the more concentrated samples (0.1 and 0.2 wt.\%) at temperature higher than $50 / 60{ }^{\circ} \mathrm{C}$ the emission intensity remained constant. The first case can be explained with a positive effect of the increased amount of amorphous phase and of the increased mobility of both the interphase and amorphous components, which, favoring PE-Pery dispersion and diffusion, prevent the formation of closely spaced quenching centers. On the other hand, the phenomenon observed for the more concentrated samples can be related to the behavior of the interphase. When, at temperature higher than $60^{\circ} \mathrm{C}$, the interphase of the more concentrated samples converts to amorphous phase, PE-Pery monomers previously enclosed in the interphase move into amorphous regions, so increasing the dye concentration and the probability of formation of the quenching centers. If this concentration effect prevails on the positive effect of the increase of LLDPE mobility, the fluorescence emission of the monomer can stop increasing. 


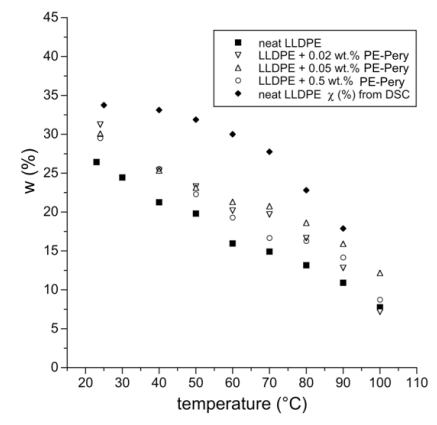

(a)

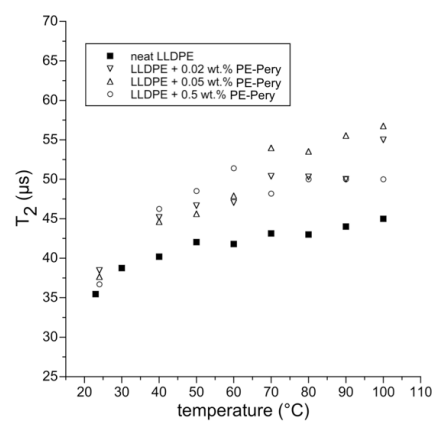

(c)

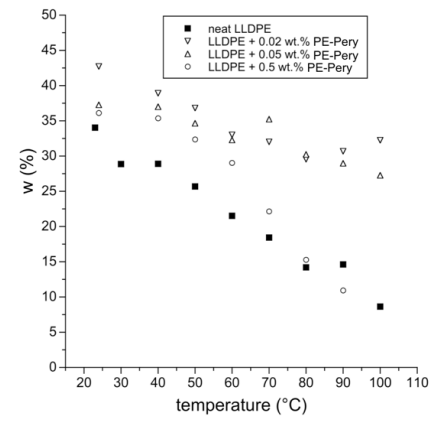

(b)

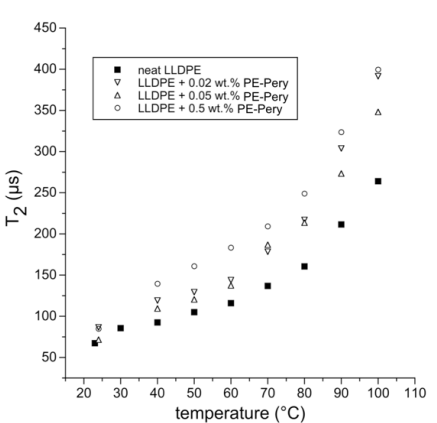

(d)
Figure 10. Results of the ${ }^{1} \mathrm{H}$ FID analysis carried out on LLDPE and LLDPE containing different amounts of PE-Pery (expressed in wt.\%) at variable temperature: (a) weight percentage of the Pake function vs. temperature; crystallinity values $(\chi(\%))$ determined by DSC are also reported (black diamonds); (b) weight percentage of the Gaussian function vs. temperature; (c) $T_{2}$ of the Gaussian function vs. temperature; (d) $T_{2}$ of the exponential function vs. temperature.

\section{Conclusions}

We have demonstrated that a fluorescent dye, namely PEPery, characterized by aggregachromic features, once embedded into LLDPE, confers thermochromic characteristics to the resulting films. Fluorescence emission of the monomeric form of PE-Pery in LLDPE was characterized by two main bands at 525 and $565 \mathrm{~nm}$, which resulted progressively quenched with increasing concentration in favor of a broad and unstructured band at $620-630 \mathrm{~nm}$, attributed to the PE-Pery aggregates. The fluorescence intensity of films with increasing temperature from 30 to $70{ }^{\circ} \mathrm{C}$ showed a specific trend concerning the monomer emission at $525 \mathrm{~nm}$ with a steeper variation in going from 40 to $55^{\circ} \mathrm{C}$. Conversely, the intensity of the red-shifted aggregates band did not change as much as the monomer intensity, thus suggesting that the thermochromic behaviour could not be only addressed to PE-Pery disaggregation. DSC and SSNMR investigations suggested that the fluorescence intensity recovery of LLDPE films with temperature might be caused by an increase of the amorphous content and polymer mobility in the $40-60{ }^{\circ} \mathrm{C}$ range. More specifically, this phenomenon is governed by the monomers of PE-Pery remained trapped at the interphase during film formation. Upon increasing temperature, the mobility of both the interphase and amorphous phase increases, allowing dye molecules dispersion and diffusion and therefore favouring their emission.

In light of this peculiar response, PE-Pery-enriched LLDPE films respond to temperature variations close to the physiological regime, providing reversible color changes from red-violet to yellow-green.

\section{Acknowledgements}

This work was partially supported by the Fondazione di Pisa under "POLOPTEL" project no. 167/09 and by MIUR-PRIN (2010XLLNM3).

\section{Notes and references}

1. O. S. Wolfbeis, J. Mater. Chem., 2005, 15, 2657-2669.

2. D. Ding, K. Li, B. Liu and Z. Tang Ben, Accounts of chemical research, 2013, 46, 2441-2453.

3. R. Hu, N. L. C. Leung and B. Z. Tang, Chemical Society Reviews, 2014, 43, 4494-4562.

4. J. Mei, Y. Hong, J. W. Y. Lam, A. Qin, Y. Tang and B. Z. Tang, Advanced Materials (Weinheim, Germany), 2014, 26, 54295479.

5. M. C. Moreno-Bondi, O. S. Wolfbeis, M. J. P. Leiner and B. P. H. Schaffar, Analytical Chemistry, 1990, 62, 2377-2380.

6. A. Mills, Q. Chang and N. McMurray, Analytical Chemistry, 1992, 64, 1383-1389.

7. G. Martini, E. Martinelli, G. Ruggeri, G. Galli and A. Pucci, Dyes and Pigments, 2015, 113, 47-54.

8. I. Platonova, A. Branchi, M. Lessi, G. Ruggeri, F. Bellina and A. Pucci, Dyes and Pigments, 2014, 110, 249-255.

9. P. Minei, M. Koenig, A. Battisti, M. Ahmad, V. Barone, T. Torres, D. M. Guldi, G. Brancato, G. Bottari and A. Pucci, Journal of Materials Chemistry C, 2014, 2, 9224-9232.

10. P. Minei, A. Battisti, S. Barondi, M. Lessi, F. Bellina, G. Ruggeri and A. Pucci, ACS Macro Letters, 2013, 2, 317-321.

11. F. Ciardelli, G. Ruggeri and A. Pucci, Chemical Society Reviews, 2013, 42, 857-870.

12. A. Pucci and G. Ruggeri, J. Mater. Chem., 2011, 21, 8282-8291.

13. A. Pucci, R. Bizzarri and G. Ruggeri, Soft Matter, 2011, 7, 36893700.

14. G. Prampolini, F. Bellina, M. Biczysko, C. Cappelli, L. Carta, M. Lessi, A. Pucci, G. Ruggeri and V. Barone, Chemistry--A European Journal, 2013, 19, 1996-2004.

15. M. M. Caruso, D. A. Davis, Q. Shen, S. A. Odom, N. R. Sottos, S. R. White and J. S. Moore, Chemical Reviews, 2009, 109, 57555798.

16. A. Pucci, T. Biver, G. Ruggeri, L. Itzel Meza and Y. Pang, Polymer, 2005, 46, 11198-11205.

17. A. Seeboth, D. Loetzsch, R. Ruhmann and O. Muehling, Chemical Reviews, 2014, 114, 3037-3068.

18. A. Seeboth and D. Loetzsch, Encyclopedia of Polymer Science and Technology (4th Edition), 2014, 14, 38-60.

19. A. Seeboth, J. Kriwanek and R. Vetter, J. Mater. Chem., 1999, 9, 2277-2278.

20. A. Seeboth, D. Loetzsch and R. Ruhmann, Journal of Materials Chemistry C: Materials for Optical and Electronic Devices, 2013, 1, 2811-2816. 
21. C. E. Sing, J. Kunzelman and C. Weder, J. Mater. Chem. , 2009, 19, 104-110.

22. J. Kunzelman, T. Chung, P. T. Mather and C. Weder, J. Mater. Chem. , 2008, 18, 1082-1086.

23. B. R. Crenshaw, J. Kunzelman, C. E. Sing, C. Ander and C. Weder, Macromolecular Chemistry and Physics, 2007, 208, 572-580.

24. M. Kinami, B. R. Crenshaw and C. Weder, Chem. Mater. , 2006, 18, 946-955.

25. P. Bamfield and M. G. Hutchings, Chromic Phenomena: Technological Applications of Colour Chemistry, Second Edition, RSC Publishing, Cambridge, UK, 2nd edn., 2010.

26. B. Valeur and M. N. Berberan-Santos, Molecular Fluorescence: Principles and Applications, Wiley- $\mathrm{VCH}$, Weinheim (Germany), second edn., 2013.

27. B. R. Crenshaw and C. Weder, Adv. Mater. , 2005, 17, 14711476.

28. J. Kunzelman, B. R. Crenshaw, M. Kinami and C. Weder, Macromol. Rapid Commun. , 2006, 27, 1981-1987.

29. F. Martini, S. Borsacchi, M. Geppi, G. Ruggeri and A. Pucci, Polymer Chemistry, 2014, 5, 828-835.

30. A. Pucci, F. Signori, R. Bizzarri, S. Bronco, G. Ruggeri and F. Ciardelli, J. Mater. Chem., 2010, 20, 5843-5852.

31. F. Donati, A. Pucci, L. Boggioni, I. Tritto and G. Ruggeri, Macromolecular Chemistry and Physics, 2009, 210, 728-735.

32. A. Pucci, F. Donati, S. Nazzi, G. U. Barretta, G. Pescitelli, L. Di Bari and G. Ruggeri, React. Funct. Polym., 2010, 70, 951-960.

33. I. S. Park, H. J. Park and J.-M. Kim, ACS Applied Materials \& Interfaces, 2013, 5, 8805-8812.

34. Z. Yuan, C.-W. Lee and S.-H. Lee, Angewandte Chemie, 2004, 116, 4293-4296.

35. S. Borsacchi, U. Sudhakaran, M. Geppi, L. Ricci, V. Liuzzo and G. Ruggeri, Langmuir, 2013, 29, 9164-9172.

36. M. Geppi, S. Borsacchi and G. Mollica, Encyclopedia of NMR, 2012, 8, 4619-4627.
37. F. Donati, A. Pucci, C. Cappelli, B. Mennucci and G. Ruggeri, J. Phys. Chem. B 2008, 112, 3668-3679.

38. F. Ammer, A. Penzkofer and P. Weidner, Chemical Physics, 1995, 192, 325-331.

39. M. Wittmann and A. Penzkofer, Chemical Physics, 1993, 172, 339-348.

40. Y. Lu and A. Penzkofer, Chemical Physics, 1986, 107, 175-184.

41. J. Brandrup, E. H. Immergut and E. A. Grulke, eds., Polymer Handbook, John Wiley \& Sons, Inc., New York, 1999.

42. P. J. Flory, Journal of the American Chemical Society, 1962, 84, 2857-2867.

43. D. Dadayli, R. K. Harris, A. M. Kenwright, B. J. Say and M. M. Sunnetcioglu, Polymer, 1994, 35, 4083-4087.

44. E. W. Hansen, P. E. Kristiansen and B. r. Pedersen, The Journal of Physical Chemistry B, 1998, 102, 5444-5450.

45. S. Borsacchi, S. Cappellozza, D. Catalano, M. Geppi and V. lerardi, Biomacromolecules, 2006, 7, 1266-1273.

46. K. Schaler, E. Ostas, K. Schroter, T. Thurn-Albrecht, W. H. Binder and K. SaalwV§chter, Macromolecules (Washington, DC, United States), 2011, 44, 2743-2754.

47. R. Kitamaru, F. Horii and S. H. Hyon, Journal of Polymer Science: Polymer Physics Edition, 1977, 15, 821-836.

48. K. Schmidt-Rohr, J. Clauss and H. W. Spiess, Macromolecules, 1992, 25, 3273-3277.

49. T. G. Fox and P. J. Flory, J. Appl. Phys., 1950, 21, 581. 